\title{
The Nehari manifold approach for multiplicity of positive solutions to semilinear elliptic system involving multi-singular inverse square potentials with Sobolev critical exponent
}

\author{
A. Akhavan ${ }^{1}$ H. Rahimi ${ }^{1}$
}

Received: 12 March 2017 / Accepted: 10 July 2017/Published online: 14 August 2017

(c) The Author(s) 2017. This article is an open access publication

\begin{abstract}
Using the Nehari manifold and variational methods, the existence and multiplicity of positive solutions for the multi-singular semilinear elliptic system with critical growth terms in bounded domains are investigated. In addition, under appropriate assumptions, it is shown that the system has at least two positive solutions when the pair of the parameters $(\alpha, \beta)$ belongs to a certain subset of $\mathbb{R}^{2}$.
\end{abstract}

Keywords Nehari manifold · Multi-singular · Sobolev critical exponent

\section{Introduction}

In this paper, we consider the following semilinear elliptic system:

$$
\left\{\begin{array}{l}
-\Delta z-\sigma \frac{z}{|x|^{2}}=\frac{1}{2^{*}} F_{z}(x, z, y)+\alpha g(x)|z|^{q-2} z \\
-\Delta y-\sigma \frac{y}{|x|^{2}}=\frac{1}{2^{*}} F_{y}(x, z, y)+\beta h(x)|y|^{q-2} y \\
z>0, y>0 \\
z=y=0
\end{array}\right.
$$

in $\Omega$,

in $\Omega$, in $\Omega$, on $\partial \Omega$.

where $0 \in \Omega \subset \mathbb{R}^{N}(N \geq 3)$ is a bounded domain with smooth boundary $\partial \Omega$, such that $0 \leq \sigma<\bar{\sigma}:=\left(\frac{N-2}{2}\right)^{2}$, and

H. Rahimi

rahimi@iauctb.ac.ir

1 Department of Mathematics, Faculty of Science, Central Tehran Branch, Islamic Azad University, Tehran, Iran
$F \in C^{1}\left(\bar{\Omega} \times\left(\mathbb{R}^{+}\right)^{2}, \mathbb{R}^{+}\right)$is positively homogeneous of degree $2^{*}\left(2^{*}:=\frac{2 N}{N-2}\right.$ denotes the critical Sobolev exponent), that is, $F(x, t z, t y)=t^{2^{*}} F(x, z, y)(t>0)$ holds for all $(x, z, y) \in \bar{\Omega} \times\left(\mathbb{R}^{+}\right)^{2}$, with $1 \leq q<2, \alpha>0, \sigma>0$.

We use $H_{0}^{1}(\Omega)$ to denote the completion of $C_{0}^{\infty}(\Omega)$ with respect to the norm:

$$
\|z\|_{H_{0}^{1}(\Omega)}=\left(\int_{\Omega}\left(|\nabla z|^{2}-\sigma \frac{z^{2}}{|x|^{2}}\right) \mathrm{d} x\right)^{\frac{1}{2}} .
$$

In the Banach space $Q=H_{0}^{1}(\Omega) \times H_{0}^{1}(\Omega)$, we introduce the norm:

$$
\|(z, y)\|_{Q}=\left(\int_{\Omega}\left(|\nabla z|^{2}+|\nabla y|^{2}-\sigma \frac{z^{2}+y^{2}}{|x|^{2}}\right) \mathrm{d} x\right)^{\frac{1}{2}} .
$$

We set $\omega_{0}=\left(\frac{q}{2}\right) \omega$, where

$$
\begin{aligned}
& \omega=\left(\frac{2-q}{2^{*}-q}\right)^{\frac{2}{2^{*}-2}}\left(\frac{2^{*}-q}{2^{*}-2}|\Omega|^{\frac{2^{*}-q}{2^{*}}}\right)^{-\frac{2}{2-q}} S_{\sigma}^{\frac{q}{2-q}} I_{F}^{\frac{N}{2}}, \\
& \Theta_{\xi}=\left\{(\alpha, \beta) \in \mathbb{R}^{+} \times \mathbb{R}^{+} \mid 0<\left(\alpha\left|g^{+}\right|_{\infty}\right)^{\frac{2}{2-q}}+\left(\beta\left|h^{+}\right|_{\infty}\right)^{\frac{2}{2-q}}<\xi\right\} .
\end{aligned}
$$

fore starting our result, we need the following assumptions:

$\left(F_{1}\right) \quad F: \bar{\Omega} \times \mathbb{R}^{+} \rightarrow \mathbb{R}^{+}$is a $C^{1}$ function, such that $F(x, t z, t y)=t^{2^{*}} F(x, z, y)$, for $t>0, \forall x \in \bar{\Omega},(z, y) \in\left(\mathbb{R}^{+}\right)^{2}$;

$\left(F_{2}\right) \quad F(x, z, 0)=F(x, 0, y)=\frac{\partial F}{\partial y}(x, 0, y)=\frac{\partial F}{\partial z}(x, z, 0)$, where $z, y \in \mathbb{R}^{+}$;

$\left(F_{3}\right) \quad \frac{\partial F}{\partial z}(x, z, y), \frac{\partial F}{\partial y}(x, z, y)$, are strictly increasing functions about $z$ and $y$ for all $z>0, y>0$.

Remark 1.1 We point out some important properties of homogeneous functions. Let $\kappa \geq 1$ and $H$ be a differentiable $\kappa$-homogeneous function, then 
(i) for all $m, n \in \mathbb{R}, m H_{m}(m, n)+n H_{n}(m, n)=$ $\kappa H(m, n)$;

(ii) there exists $M_{H}>0$, such that $|H(m, n)|$ $\leq M_{H}\left(|m|^{\kappa}+|n|^{\kappa}\right)$, for all $m, n \in \mathbb{R}$, where $M_{H}=\max \left\{H(m, n): m, n \in \mathbb{R},|m|^{\kappa}+|n|^{\kappa}=1\right\}$;

(iii) the maximum $M_{H}$ is attained for some $\left(m_{0}, n_{0}\right) \in \mathbb{R}^{2}$, i.e., $\left|m_{0}\right|^{\kappa}+\left|n_{0}\right|^{\kappa}=1$ and $H$ $\left(m_{0}, n_{0}\right)=M_{H}$;

(iv) $H_{m}(m, n), H_{n}(m, n)$ are $(\kappa-1)$ homogeneous.

By $\left(F_{1}\right)$ and the properties of homogeneous functions, we have

$F_{z}(z, y) z+F_{y}(z, y) y=2^{*} F(z, y)$,

and

$F(z, y) \leq\left(M_{F}\left(|z|^{2}+|y|^{2}\right)\right)^{\frac{2^{*}}{2}}$,

where

$M_{F}=\max \left\{(F(z, y))^{\frac{2^{*}}{2}}:(z, y) \in \mathbb{R}^{2},|z|^{2}+|y|^{2}=1\right\}$.

Define

$I_{F}:=\inf _{(z, y) \in Q}\left\{\frac{\|(z, y)\|^{2}}{\left(\int_{\Omega} F(x, z, y) \mathrm{d} x\right) \frac{2}{2^{*}}}: \int_{\Omega} F(x, z, y) \mathrm{d} x>0\right\}$,

then we have [1]

$I_{F}=M_{F}^{-1} S_{\sigma}$.

The main result of this paper is the following theorems.

Theorem 1.2 Assume that parameters $(\alpha, \beta) \in \Theta_{\omega}$ and $\left(F_{1}\right)-\left(F_{3}\right)$ hold. Then, problem (1) has at lest one positive solutions.

Theorem 1.3 Assume that parameters $(\alpha, \beta) \in \Theta_{\omega_{0}^{*}}$ and $\left(F_{1}\right)-\left(F_{3}\right)$ hold. Then, problem (1) has at lest two positive solutions.

This paper is organized as follows. In Sect. 2, we consider some properties of Nehari manifold. In Sect. 3, we show that $(\mathrm{PS})_{c}$ condition holds for $E_{(\alpha, \beta)}$ with $c$ in certain interval, and then, we prove Theorems 1.2 and 1.3.

\section{Nehari manifold}

Definition 2.1 A pair of functions $(z, y) \in Q$ is weak solution to (1), if for all $(\phi, \varphi) \in Q$ :

$$
\begin{aligned}
& \int_{\Omega}(\nabla z \nabla \phi+\nabla y \nabla \varphi) \mathrm{d} x-\int_{\Omega} \frac{\sigma}{|x|^{2}}(z \phi+y \varphi) \mathrm{d} x \\
& -\frac{1}{2^{*}} \int_{\Omega} \frac{\partial F(x, z, y)}{\partial z} \phi \mathrm{d} x \\
& -\frac{1}{2^{*}} \int_{\Omega} \frac{\partial F(x, z, y)}{\partial y} \varphi \mathrm{d} x-\alpha \int_{\Omega} g(x)|z|^{q-2} \phi \mathrm{d} x \\
& -\beta \int_{\Omega} h(x)|y|^{q-2} y \varphi \mathrm{d} x=0 .
\end{aligned}
$$

Thus, the corresponding energy functional of problem (1) is defined by

$E_{\alpha, \beta}(z, y)=\frac{1}{2}\|(z, y)\|_{Q}^{2}-\frac{1}{2^{*}} \int_{\Omega} F(x, z, y) \mathrm{d} x-\frac{1}{q} D_{\alpha, \beta}(z, y)$,

for $(z, y) \in Q$, where $D_{\alpha, \beta}(z, y)=\alpha \int_{\Omega} g(x)|z|^{q} \mathrm{~d} x+\beta \int_{\Omega} h$ $(x)|y|^{q} \mathrm{~d} x$.

Throughout of this paper for $0 \leq \sigma<\bar{\sigma}$, we suppose that $S_{\mu}$ is the best Sobolev embedding constant [5, 6], where $S_{\sigma}$ is independent of $\Omega$ [9].

It is well known that the weak solutions of (1) are the critical points of the energy functional $E_{\alpha, \beta}$. If $E_{\alpha, \beta}$ is bounded below and has a minimizer on $Q$, then this minimizer is a critical point of $E_{\alpha, \beta}$. Therefore, it is a solution of the corresponding elliptic system. However, the energy function, $E_{\alpha, \beta}$, is not bounded below on the whole space $Q$, but is bounded on an appropriate subset, that is called Nehari manifold. Then, we have

$\mathcal{N}_{\alpha, \beta}=\left\{(z, y) \in Q:\left\langle E_{\alpha, \beta}^{\prime}(z, y),(z, y)\right\rangle=0\right\}$.

Thus, $(z, y) \in \mathcal{N}_{\alpha, \beta}$ if and only if

$$
\left\langle E_{\lambda, \beta}^{\prime}(z, y),(z, y)\right\rangle=\|(z, y)\|_{Q}^{2}-\int_{\Omega} F(x, z, y) \mathrm{d} x-D_{\alpha, \beta}(z, y)=0 .
$$

Note that $\mathcal{N}_{\alpha, \beta}$ contains every non-zero solution of Eq. (1). We now recall the following Lemma.

Lemma 2.2 [2, Lemma 2.1] The energy functional $E_{\alpha, \beta}$ is coercive and bounded below on $\mathcal{N}_{\alpha, \beta}$.

Now, we split $\mathcal{N}_{\alpha, \beta}$ into three parts:

$\mathcal{N}_{\alpha, \beta}^{+}=\left\{(z, y) \in \mathcal{N}_{\alpha, \beta}:\left\langle\psi_{\alpha, \beta}^{\prime}(z, y),(z, y)\right\rangle>0\right\}$
$\mathcal{N}_{\alpha, \beta}^{0}=\left\{(z, y) \in \mathcal{N}_{\alpha, \beta}:\left\langle\psi_{\alpha, \beta}^{\prime}(z, y),(z, y)\right\rangle=0\right\}$
$\mathcal{N}_{\alpha, \beta}^{-}=\left\{(z, y) \in \mathcal{N}_{\alpha, \beta}:\left\langle\psi_{\alpha, \beta}^{\prime}(z, y),(z, y)\right\rangle<0\right\}$.

By an argument similar to that [4, Theorem 2.3], we have the following lemma. 
Lemma 2.3 Assume that $\left(z_{0}, y_{0}\right)$ is local minimizer for $E_{\alpha, \beta}$ on $\mathcal{N}_{\alpha, \beta}$ and $\left(z_{0}, y_{0}\right) \notin \mathcal{N}_{\alpha, \beta}^{0}$. Then, $E_{\alpha, \beta}^{\prime}\left(z_{0}, y_{0}\right)=0$ in $Q^{-1}$.

Lemma 2.4 There exists a positive number $\omega=$ $\omega\left(q, N, M_{F}, S,|\Omega|\right)$ such that if $(\alpha, \beta) \in \Theta_{\omega}$, then $\mathcal{N}_{\alpha, \beta}^{0}=\emptyset$.

Using Lemma 2.4, we have $\mathcal{N}_{\alpha, \beta}=\mathcal{N}_{\alpha, \beta}^{+} \cup \mathcal{N}_{\alpha, \beta}^{-}$. Define

$$
\begin{aligned}
\vartheta_{\alpha, \beta} & =\inf _{(z, y) \in \mathcal{N}_{\alpha, \beta}} E_{\alpha, \beta}(z, y) ; \vartheta_{\alpha, \beta}^{+} \\
& =\inf _{(z, y) \in \mathcal{N}_{\alpha, \beta}^{+}} E_{\alpha, \beta}(z, y) ; \vartheta_{\alpha, \beta}^{-}=\inf _{(z, y) \in \mathcal{N}_{\alpha, \beta}^{-}} E_{\alpha, \beta}(z, y) .
\end{aligned}
$$

Now, we recall the following results.

Proposition 2.5 [2, Theorem 2.5]

(i) If $(\alpha, \beta) \in \Theta_{\omega}$, then $\vartheta_{\alpha, \beta} \leq \vartheta_{\alpha, \beta}^{+}<0$;

(ii) If $(\alpha, \beta) \in \Theta_{\omega_{0}}$, then $\vartheta_{\alpha, \beta}^{-}>c_{0}$ for some $c_{0}=c_{0}(\alpha, \beta, q, N, S,|\Omega|)>0$.

Lemma 2.6 [2, Lemma 2.6] If $\int_{\Omega} F(x, z, y) \mathrm{d} x>0$, then there are unique $t^{+}$and $t^{-}$with $0<t^{+}<t_{\max }<t^{-}$, such that $\left(t^{+} z, t^{+} y\right) \in \mathcal{N}_{\alpha, \beta}^{+},\left(t^{-} z, t^{-} y\right) \in \mathcal{N}_{\alpha, \beta}^{-}($for each $(z, y) \in Q)$ and

$$
\begin{aligned}
E_{\alpha, \beta}\left(t^{+} z, t^{+} y\right) & =\inf _{0 \leq t \leq t_{\max }} E_{\alpha, \beta}(t z, t y), \quad E_{\alpha, \beta}\left(t^{-} z, t^{-} y\right) \\
& =\sup _{t \geq 0} E_{\alpha, \beta}(t z, t y) .
\end{aligned}
$$

\section{Proof of Theorems 1.2 and 1.3}

This section is devoted to providing of the main results of this paper. In the first, we recall some notions and results. For details, see [7, 8, 11, 13, 14].

\section{Definition 3.1}

(i) For $c \in \mathbb{R}$, a sequence $\left\{\left(z_{n}, y_{n}\right)\right\}$ is a (PS) sequence in $Q$ for $E_{\alpha, \beta}$ if $E_{\alpha, \beta}\left(z_{n}, y_{n}\right)=$ $c+o_{n}(1)$, and $E_{\alpha, \beta}^{\prime}\left(z_{n}, y_{n}\right)=o_{n}(1)$ strongly in $Q$, as $n \rightarrow \infty$.

(ii) $\quad c \in \mathbb{R}$ is a $(\mathrm{PS})_{c}$ value in $Q$ for $E_{\alpha, \beta}$ if there exists a $(\mathrm{PS})_{c}$ sequence in $Q$ for $E_{\alpha, \beta}$.

(iii) $E_{\alpha, \beta}$ satisfies the (PS $)_{c}$ condition in $Q$ if any $(\mathrm{PS})_{c}$ sequence $\left\{\left(z_{n}, y_{n}\right)\right\}$ in $Q$ for $E_{\alpha, \beta}$ contains a convergent subsequence.

We also need the following theorem due to Tsung-Fang $\mathrm{Wu}[15]$.

Lemma 3.2 [15, Proposition 9]

(i) If $(\alpha, \beta) \in \Theta_{\omega}$, then there exists a $(\mathrm{PS})_{\vartheta_{\alpha, \beta}}$ sequence $\left\{\left(z_{n}, y_{n}\right)\right\} \subset \mathcal{N}_{\alpha, \beta}$ in $Q$ for $E_{\alpha, \beta}$. (ii) If $(\alpha, \beta) \in \Theta_{\omega_{0}}$, then there exists a (PS $)_{\vartheta_{\alpha, \beta}^{-}}$ sequence $\left\{\left(z_{n}, y_{n}\right)\right\} \subset \mathcal{N}_{\alpha, \beta}^{-}$in $Q$ for $E_{\alpha, \beta}$.

In the following theorem, we express the existence of a local minimum for $E_{\alpha, \beta}$ on $\mathcal{N}_{\alpha, \beta}^{+}$.

Theorem 3.3 Assume that $(\alpha, \beta) \in \Theta_{\omega}$, and $\left(F_{1}\right)-\left(F_{3}\right)$ hold. Then, $E_{\alpha, \beta}$ has a minimizer $\left\{\left(z_{0}^{+}, y_{0}^{+}\right)\right\} \subset \mathcal{N}_{\alpha, \beta}^{+}$and

(i) $E_{\alpha, \beta}\left(z_{0}^{+}, y_{0}^{+}\right)=\vartheta_{\alpha, \beta}=\vartheta_{\alpha, \beta}^{+}$,

(ii) $\left(z_{0}^{+}, y_{0}^{+}\right)$is a positive solution of problem (1).

Proof Using Lemma 3.2(i), there exists a minimizing sequence $\left\{\left(z_{n}, y_{n}\right)\right\}$ for $E_{\alpha, \beta}$ on $\mathcal{N}_{\alpha, \beta}$, such that

$$
E_{\alpha, \beta}\left(z_{n}, y_{n}\right)=\vartheta_{\alpha, \beta}+o(1) \text { and } E_{\alpha, \beta}^{\prime}\left(z_{n}, y_{n}\right)=o(1)\left(\text { in } \mathrm{Q}^{-1}\right) \text {. }
$$

Then, by Lemma 2.2 and the compact imbedding theorem, there exist a subsequence $\left\{\left(z_{n}, y_{n}\right)\right\}$ and $\left\{\left(z_{0}^{+}, y_{0}^{+}\right)\right\} \in Q$, such that

$\begin{cases}z_{n} \rightarrow z_{0}^{+} & \text {weakly in } \mathrm{Q}_{0}^{1, \mathrm{p}}, \\ z_{n} \rightarrow z_{0}^{+} & \text {strongly in } \mathrm{L}^{\mathrm{q}}(\Omega), \\ y_{n} \rightarrow y_{0}^{+} & \text {weakly in } \mathrm{Q}_{0}^{1, \mathrm{p}}, \\ y_{n} \rightarrow y_{0}^{+} & \text {strongly in } \mathrm{L}^{\mathrm{q}}(\Omega) .\end{cases}$

This implies that $D_{\alpha, \beta}\left(z_{n}, y_{n}\right) \rightarrow D_{\alpha, \beta}\left(z_{0}^{+}, y_{0}^{+}\right)$as $n \rightarrow \infty$.

Using (6) and (7), it is not hard to see that $\left(z_{0}^{+}, y_{0}^{+}\right)$is a weak solution of (1).

Since

$$
\begin{aligned}
E_{\alpha, \beta}\left(z_{n}, y_{n}\right) & =\frac{1}{N}\left\|\left(z_{n}, y_{n}\right)\right\|^{2}-\frac{2^{*}-q}{2^{*} q} D_{\alpha, \beta}\left(z_{n}, y_{n}\right) \\
& \geq-\frac{2^{*}-q}{2^{*} q} D_{\alpha, \beta}\left(z_{n}, y_{n}\right)
\end{aligned}
$$

and using Lemma 2.5(i)

$$
E_{\alpha, \beta}\left(z_{n}, y_{n}\right) \rightarrow \vartheta_{\alpha, \beta}^{+}<0 \quad \text { as } \quad n \rightarrow \infty
$$

Letting $n \rightarrow \infty$, we see that $D_{\alpha, \beta}\left(z_{0}^{+}, y_{0}^{+}\right)>0$. Thus, $\left(z_{0}^{+}, y_{0}^{+}\right)$is a nontrivial solution of problem (1).

Now, it follows that $z_{n} \rightarrow z_{0}^{+}$strongly in $H_{0}^{1}(\Omega), y_{n} \rightarrow$ $y_{0}^{+}$strongly in $H_{0}^{1}(\Omega)$ and $E_{\alpha, \beta}\left(z_{0}^{+}, y_{0}^{+}\right)=\vartheta_{\alpha, \beta}$. As $\left(z_{n}, y_{n}\right) \in$ $\mathcal{N}_{\alpha, \beta}$ and applying Fatou's lemma, we have

$$
\begin{aligned}
\vartheta_{\alpha, \beta} & \leq E_{\alpha, \beta}\left(z_{0}^{+}, y_{0}^{+}\right)=\frac{1}{N}\left\|\left(z_{0}^{+}, y_{0}^{+}\right)\right\|^{2}-\frac{2^{*}-q}{2^{*} q} D_{\alpha, \beta}\left(z_{0}^{+}, y_{0}^{+}\right) \\
& \leq \liminf _{n \rightarrow \infty}\left(\frac{1}{N}\left\|\left(z_{n}, y_{n}\right)\right\|^{2}-\frac{2^{*}-q}{2^{*} q} D_{\alpha, \beta}\left(z_{n}, y_{n}\right)\right) \\
& \leq \liminf _{n \rightarrow \infty} E_{\alpha, \beta}\left(z_{n}, y_{n}\right)=\vartheta_{\alpha, \beta} .
\end{aligned}
$$


This implies that

$E_{\alpha, \beta}\left(z_{0}^{+}, y_{0}^{+}\right)=\vartheta_{\alpha, \beta} \quad$ and $\quad \lim _{n \rightarrow \infty}\left\|\left(z_{n}, y_{n}\right)\right\|^{2}=\left\|\left(z_{0}^{+}, y_{0}^{+}\right)\right\|^{2}$.

Let $\left(\bar{z}_{n}, \bar{y}_{n}\right)=\left(z_{n}, y_{n}\right)-\left(z_{0}^{+}, y_{0}^{+}\right)$, then Brézis-Lieb lemma [3], implies

$$
\left\|\left(\bar{z}_{n}, \bar{y}_{n}\right)\right\|^{2}=\left\|\left(z_{n}, y_{n}\right)\right\|^{2}-\left\|\left(z_{0}^{+}, y_{0}^{+}\right)\right\|^{2} \text {. }
$$

Therefore, $z_{n} \rightarrow z_{0}^{+}$strongly in $H_{0}^{1}(\Omega), y_{n} \rightarrow y_{0}^{+}$strongly in $H_{0}^{1}(\Omega)$. Moreover, we have $\left(z_{0}^{+}, y_{0}^{+}\right) \in \mathcal{N}_{\alpha, \beta}^{+}$. In fact, if $\left(z_{0}^{+}, y_{0}^{+}\right) \in \mathcal{N}_{\alpha, \beta}^{-}$, by Lemma 2.6, there are unique $t_{0}^{+}$and $t_{0}^{-}$, such that $\left(t_{0}^{+} z_{0}^{+}, t_{0}^{+} y_{0}^{+}\right) \in \mathcal{N}_{\alpha, \beta}^{+}$and $\left(t_{0}^{-} z_{0}^{+}, t_{0}^{-} y_{0}^{+}\right) \in \mathcal{N}_{\alpha, \beta}^{-}$. In particular, we have $t_{0}^{+}<t_{0}^{-}=1$. Since

$\frac{\mathrm{d}}{\mathrm{d} t} E_{\alpha, \beta}\left(t_{0}^{+} z_{0}^{+}, t_{0}^{+} y_{0}^{+}\right)=0 \quad$ and $\quad \frac{\mathrm{d}^{2}}{\mathrm{~d} t^{2}} E_{\alpha, \beta}\left(t_{0}^{+} z_{0}^{+}, t_{0}^{+} y_{0}^{+}\right)>0$,

there exists $t_{0}^{+}<\bar{t} \leq t_{0}^{-}$, such that $E_{\alpha, \beta}\left(t_{0}^{+} z_{0}^{+}, t_{0}^{+} y_{0}^{+}\right)<$ $E_{\alpha, \beta}\left(\bar{t} z_{0}^{+}, \bar{t} y_{0}^{+}\right)$. By Lemma 2.6

$$
\begin{aligned}
& E_{\alpha, \beta}\left(t_{0}^{+} z_{0}^{+}, t_{0}^{+} y_{0}^{+}\right)<E_{\alpha, \beta}\left(\bar{t} z_{0}^{+}, \bar{t} y_{0}^{+}\right) \\
& \quad \leq E_{\alpha, \beta}\left(t_{0}^{-} z_{0}^{+}, t_{0}^{-} y_{0}^{+}\right)=E_{\alpha, \beta}\left(z_{0}^{+}, y_{0}^{+}\right),
\end{aligned}
$$

which is a contradiction.

It follows from the maximum principle that $\left(z_{0}^{+}, y_{0}^{+}\right)$is a positive solution of problem (1).

Lemma 3.4 [12, Lemma 2.2] Let $\left\{\left(z_{n}, y_{n}\right)\right\} \subset Q$ be a $(\mathrm{PS})_{c}$ sequence for $E_{\alpha, \beta}$ with $\left(z_{n}, y_{n}\right) \rightarrow(z, y)$ in $Q$, then $E_{\alpha, \beta}^{\prime}(z, y)=0$, and there exists a constant $d_{0}=$ $d_{0}(q, N, S,|\Omega|)$, such that $E_{\alpha, \beta}(z, y) \geq-d_{0}\left(\left(\alpha\left|g^{+}\right|_{\infty}\right)^{\frac{2}{2-q}}\right.$ $\left.+\left(\beta\left|h^{+}\right|_{\infty}\right)^{\frac{2}{2-q}}\right)$.

Lemma 3.5 [12, Lemma 4.1] Let $\left\{\left(z_{n}, y_{n}\right)\right\} \subset Q$ be a $(\mathrm{PS})_{c}$ sequence for $E_{\alpha, \beta}$, then $\left(z_{n}, y_{n}\right)$ is bounded in $Q$.

Lemma 3.6 Assume that $\left(F_{1}\right)-\left(F_{3}\right)$ hold. Then, $E_{\alpha, \beta}$ satisfies the $(\mathrm{PS})_{c}$ condition with $c$ satisfying

$-\infty<c<c_{\infty}=\frac{1}{N} S_{F}^{\frac{N}{2}}-d_{0}\left(\left(\alpha\left|g^{+}\right|_{\infty}\right)^{\frac{2}{2-q}}+\left(\beta\left|h^{+}\right|_{\infty}\right)^{\frac{2}{2-q}}\right)$,

where $d_{0}$ is positive constant given in Lemma 3.4.

Proof Let $\left\{\left(z_{n}, y_{n}\right)\right\} \subset Q$ be a (PS $)_{c}$ sequence for $E_{\alpha, \beta}$ with $c \in\left(-\infty, c_{\infty}\right)$. It follows from Lemma 3.5 that $\left\{\left(z_{n}, y_{n}\right)\right\}$ is bounded in $Q$, and then $\left(z_{n}, y_{n}\right) \rightarrow(z, y)$ up to a subsequence, $(z, y)$ is a critical point of $E_{\alpha, \beta}$. Furthermore, we may assume

$\left\{\begin{array}{lll}z_{n} \rightarrow z, & y_{n} \rightarrow y & \text { in } H_{0}^{1}(\Omega), \\ z_{n} \rightarrow z, & y_{n} \rightarrow y & \text { in } L^{s}(\Omega), \quad 1 \leq s<2^{*} \\ z_{n} \rightarrow z, & y_{n} \rightarrow y & \text { a.e.on } \Omega .\end{array}\right.$

Hence, $E_{\alpha, \beta}^{\prime}(z, y)=0$, and

$D_{\alpha, \beta}\left(z_{n}, y_{n}\right)=D_{\alpha, \beta}(z, y)+o_{n}(1)$.
Let $\bar{z}_{n}=z_{n}-z, \bar{y}_{n}=y_{n}-y$. Using Brézie-Lieb lemma [3], we obtain

$$
\left\|\left(\bar{z}_{n}, \bar{y}_{n}\right)\right\|^{2}=\left\|\left(z_{n}, y_{n}\right)\right\|^{2}-\|(z, y)\|^{2}+o_{n}(1) .
$$

In addition, using an argument similar to that [10, Lemma 8]

$$
\int_{\Omega} F\left(x, \bar{z}_{n}, \bar{y}_{n}\right) \mathrm{d} x=\int_{\Omega} F\left(x, z_{n}, y_{n}\right) \mathrm{d} x-\int_{\Omega} F(x, z, y) \mathrm{d} x+o_{n}(1) .
$$

Since $E_{\alpha, \beta}\left(z_{n}, y_{n}\right)=c+o(1), E_{\alpha, \beta}^{\prime}(z, y)=o_{n}(1)$ and (9)(11), we can deduce that

$\frac{1}{2}\left\|\left(\bar{z}_{n}, \bar{y}_{n}\right)\right\|^{2}-\frac{1}{2^{*}} \int_{\Omega} F\left(x, \bar{z}_{n}, \bar{y}_{n}\right) \mathrm{d} x=c-E_{\alpha, \beta}(z, y)+o_{n}(1)$,

and

$\left\|\left(\bar{z}_{n}, \bar{y}_{n}\right)\right\|^{2}-\int_{\Omega} F\left(x, \bar{z}_{n}, \bar{y}_{n}\right) \mathrm{d} x=o_{n}(1)$.

Hence, we may assume that

$$
\left\|\left(\bar{z}_{n}, \bar{y}_{n}\right)\right\|^{2} \rightarrow l, \quad \int_{\Omega} F\left(x, \bar{z}_{n}, \bar{y}_{n}\right) \rightarrow l .
$$

If $l=0$, then the proof is complete. Assume that $l>0$, then from (14), we obtain

$$
\begin{aligned}
I_{F} l^{\frac{2}{2^{*}}} & =I_{F} \lim _{n \rightarrow \infty}\left(\int_{\Omega} F\left(x, \bar{z}_{n}, \bar{y}_{n}\right) \mathrm{d} x\right)^{\frac{2}{2^{*}}} \\
& \leq \lim _{n \rightarrow \infty}\left\|\left(\bar{z}_{n}, \bar{y}_{n}\right)\right\|^{2}=l
\end{aligned}
$$

which implies that $l \geq I_{F}^{\frac{N}{2}}$. In addition, from Lemma 3.4, relations (12) and (14), we get

$$
\begin{aligned}
c & =\left(\frac{1}{2}-\frac{1}{2^{*}}\right) l+E_{\alpha, \beta}(z, y) \\
& \geq \frac{1}{N} I_{F}^{\frac{N}{2}}-d_{0}\left(\left(\alpha\left|g^{+}\right|_{\infty}\right)^{\frac{2}{2-q}}+\left(\beta\left|h^{+}\right|_{\infty}\right)^{\frac{2}{2-q}}\right),
\end{aligned}
$$

which contradicts $c<\frac{1}{N} I_{F}^{\frac{N}{2}}-d_{0}\left(\left(\alpha\left|g^{+}\right|_{\infty}\right)^{\frac{2}{2-q}}+\left(\beta\left|h^{+}\right|_{\infty}\right)^{\frac{2}{2-q}}\right)$.

Lemma 3.7 Assume that $\left(F_{1}\right)-\left(F_{3}\right)$ hold. Then, there exist a nonnegative function $(z, y) \in Q \backslash\{(0,0)\}$ and $\omega^{*}>0$, such that for $0<\left(\alpha\left|g^{+}\right|_{\infty}\right)^{\frac{2}{2-q}}+\left(\beta\left|h^{+}\right|_{\infty}\right)^{\frac{2}{2-q}}<\omega^{*}$, we have

$\sup _{t \geq 0} E_{\alpha, \beta}(t z, t y)<c_{\infty}$

In particular, $\vartheta_{\alpha, \beta}^{-}<c_{\infty}$ for all $0<\left(\alpha\left|g^{+}\right|_{\infty}\right)^{\frac{2}{2-q}}+\left(\beta\left|h^{+}\right|_{\infty}\right)^{\frac{2}{2-q}}<\omega^{*}$.

Proof Since $0 \in \Omega$, there is $\varrho_{0}>0$, such that $B_{2 \varrho_{0}}(0) \subset \Omega$. Now, we define a cut-off function $\gamma(x)$ 
satisfying $\gamma(x)=1$ for $|x| \leq \varrho_{0}, \gamma(x)=0$ for $|x|>2 \varrho_{0}$, $0 \leq \gamma<1$ and $|\nabla \gamma| \leq C$. For $\varepsilon>0$, let

$z_{\varepsilon}(x)=\frac{\varepsilon^{\frac{N-2}{4}} \gamma(x)}{\left(\varepsilon|x|^{\tau^{\prime} / \sqrt{\bar{\sigma}}}+|x|^{\tau / \sqrt{\bar{\sigma}}}\right)^{\sqrt{\bar{\sigma}}}}$

where $\quad \bar{\sigma}=\left(\frac{N-2}{2}\right)^{2}, \tau^{\prime}=\sqrt{\bar{\sigma}}-\sqrt{\bar{\sigma}-\sigma_{k}} \quad$ and $\quad \tau=$ $\sqrt{\bar{\sigma}}+\sqrt{\bar{\sigma}-\sigma_{k}}$. Using the property (iii) of homogeneous functions, there exists $\left(e_{1}, e_{2}\right) \in \mathbb{R}^{2}$, such that $e_{1}^{2}+e_{2}^{2}=1$ and $F\left(x, e_{1}, e_{2}\right)=M_{F}^{\frac{2^{*}}{2}}$.

For $t \geq 0$, we consider $z_{0}=e_{1} z_{\varepsilon}, y_{0}=e_{2} z_{\varepsilon} \quad$ and $\left(z_{0}, y_{0}\right) \in Q$. We now define the following functions:

$$
\begin{aligned}
G(t) & :=E_{\alpha, \beta}\left(t z_{0}, t y_{0}\right)=E_{\alpha, \beta}\left(t e_{1} z_{\varepsilon}, t e_{2} z_{\varepsilon}\right) \\
& =\frac{t^{2}}{2} \int_{\Omega}\left(\left|\nabla z_{\varepsilon}\right|^{2}-\sigma \frac{\left(z_{\varepsilon}\right)^{2}}{|x|^{2}}\right)-\frac{t^{2^{*}}}{2^{*}} \int_{\Omega} F\left(x, e_{1} z_{\varepsilon}, e_{2} z_{\varepsilon}\right) \mathrm{d} x \\
& -\frac{t^{q}}{q}\left(\alpha e_{1}^{q}+\beta e_{2}^{q}\right) \int_{\Omega}\left|z_{\varepsilon}\right|^{q}
\end{aligned}
$$

and

$\bar{G}(t):=\frac{t^{2}}{2} \int_{\Omega}\left(\left|\nabla z_{\varepsilon}\right|^{2}-\sigma \frac{\left(z_{\varepsilon}\right)^{2}}{|x|^{2}}\right)-\frac{t^{2^{*}}}{2^{*}} \int_{\Omega} F\left(x, e_{1} z_{\varepsilon}, e_{2} z_{\varepsilon}\right) \mathrm{d} x$

Step 1 We need to show that

$\sup _{t \geq 0} \bar{g}(t) \leq c_{\infty}$.

To do this, we need to established the following estimates (as $\varepsilon \rightarrow 0$ ):

$$
\begin{aligned}
& \left(\int_{\Omega}\left|z_{\varepsilon}\right|^{2^{*}} \mathrm{~d} x\right)^{\frac{2}{2^{*}}}=\left(\int_{\Omega}|U|^{2^{*}} \mathrm{~d} x\right)^{\frac{2}{2^{*}}}+o\left(\varepsilon^{\frac{N}{2}}\right), \\
& \int_{\Omega}\left(\left|\nabla z_{\varepsilon}\right|^{2}-\sigma \frac{\left(z_{\varepsilon}\right)^{2}}{|x|^{2}}\right) \mathrm{d} x=\int_{\Omega}\left(|\nabla U|^{2}-\sigma \frac{U^{2}}{|x|^{2}}\right) \mathrm{d} x+o\left(\varepsilon^{\frac{N-2}{2}}\right),
\end{aligned}
$$

$$
\frac{\int_{\Omega}\left(\left|\nabla z_{\varepsilon}\right|^{2}-\sigma \frac{\left(z_{\varepsilon}\right)^{2}}{|x|^{2}}\right) \mathrm{d} x}{\left(\int_{\Omega}\left|z_{\varepsilon}\right|^{2^{*}} \mathrm{~d} x\right)^{\frac{2}{2^{*}}}}=S_{\sigma}+o\left(\varepsilon^{\frac{N-2}{2}}\right),
$$

where $\quad U(x)=\left(|x|^{\tau^{\prime} / \sqrt{\bar{\sigma}}}+|x|^{\tau / \sqrt{\bar{\sigma}}}\right)^{-\sqrt{\bar{\sigma}}}, \quad$ and $\quad \omega_{N}=$ $2 \pi^{N / 2} / N \Gamma\left(\frac{N}{2}\right)$ is the volume of the unit ball $B(0,1)$ in $\mathbb{R}^{N}$. We only show that the equality (15) is valid. By definition of $z_{\varepsilon}$, we have

$$
\int_{\mathbb{R}^{N}}\left|z_{\varepsilon}\right|^{2^{*}} \mathrm{~d} x=\int_{\mathbb{R}^{N}} \frac{\varepsilon^{\frac{N}{2}} \gamma^{2^{*}}(x)}{\left(\varepsilon|x|^{\tau_{k}^{\prime} / \sqrt{\bar{\sigma}}}+|x|^{\tau_{k} / \sqrt{\bar{\sigma}}}\right)^{N}} \mathrm{~d} x .
$$

On the other hand, let $x=\varepsilon^{\frac{\sqrt{\sigma}}{\tau_{k}-\tau_{k}^{\prime}}}$, we can deduce that

$$
\begin{aligned}
& \left(\int_{\mathbb{R}^{N}} \frac{1}{\left(\varepsilon|x|^{\tau_{k}^{\prime} / \sqrt{\bar{\sigma}}}+|x|^{\tau_{k} / \sqrt{\bar{\sigma}}}\right)^{N}} \mathrm{~d} x\right) \\
& \quad=\varepsilon^{-\frac{N}{2}} \int_{\mathbb{R}^{N}} \frac{1}{\left(|y|^{\tau_{k}^{\prime} / \sqrt{\bar{\sigma}}}+|y|^{\tau_{k} / \sqrt{\bar{\sigma}}}\right)^{N}} \mathrm{~d} y \\
& \quad=\varepsilon^{-\frac{N}{2}}|U|_{L^{2 *}\left(\mathbb{R}^{N}\right) .}
\end{aligned}
$$

We have

$$
\begin{aligned}
0 & \leq \varepsilon^{-\frac{N}{2}}|U|_{L^{2^{*}}\left(\mathbb{R}^{N}\right)}^{2^{*}}-\varepsilon^{-\frac{N}{2}}\left|z_{\varepsilon}\right|_{2^{*}}^{2^{*}} \\
& =\int_{\mathbb{R}^{N} \backslash B\left(0, \varepsilon_{0}\right)} \frac{1}{\left(\varepsilon|x|^{\tau_{k}^{\prime} / \sqrt{\bar{\sigma}}}+|x|^{\tau / \sqrt{\bar{\sigma}}}\right)^{N}} \mathrm{~d} x \\
& \leq \int_{\mathbb{R}^{N} \backslash B\left(0, \varepsilon_{0}\right)} \frac{\mathrm{d} x}{|x|^{2^{*} \tau}} \\
& =\frac{N-2}{2} \omega_{N}\left(\varepsilon_{0}\right)^{-\frac{2 N}{N-2} \beta_{k}} \\
& \leq d_{1}=\text { Constant. }
\end{aligned}
$$

Hence

$0 \leq 1-\left|z_{\varepsilon}\right|_{L^{2^{*}}\left(\mathbb{R}^{N}\right)}^{2^{*}}|U|_{L^{2^{*}}\left(\mathbb{R}^{N}\right)}^{-2^{*}} \leq d_{1} \varepsilon^{\frac{N}{2}}|U|_{L^{2^{*}}\left(\mathbb{R}^{N}\right)}^{-2^{*}}$

that is

$$
1-d_{1} \varepsilon^{\frac{N}{2}}|U|_{L^{2^{*}}\left(\mathbb{R}^{N}\right)}^{-2^{*}} \leq\left|z_{\varepsilon}\right|_{L^{2^{*}}\left(\mathbb{R}^{N}\right)}^{2^{*}}|U|_{L^{2^{*}}\left(\mathbb{R}^{N}\right)}^{-2^{*}} \leq 1,
$$

Now, let $\varepsilon$ be small enough, such that $d_{1} \varepsilon^{\frac{N}{2}}|U|_{L^{2^{*}}\left(\mathbb{R}^{N}\right)}^{-2^{*}}<1$, then from (21), we can deduce that

$$
\begin{aligned}
& 1-d_{1} \varepsilon^{\frac{N}{2}}|U|_{L^{2^{*}}\left(\mathbb{R}^{N}\right)}^{-2^{*}} \leq\left(1-d_{1} \varepsilon^{\frac{N}{\varepsilon^{2}}}|U|_{L^{2^{*}}\left(\mathbb{R}^{N}\right)}^{-2^{*}}\right)^{\frac{2}{2^{*}}} \\
& \quad \leq\left|z_{\varepsilon}\right|_{L^{2^{*}}\left(\mathbb{R}^{N}\right)}^{2}|U|_{L^{2^{*}}\left(\mathbb{R}^{N}\right)}^{-2} \leq 1,
\end{aligned}
$$

which yield that

$$
|U|_{L^{2^{*}}\left(\mathbb{R}^{N}\right)}^{2}-d_{1} \varepsilon^{\frac{N}{2}}|U|_{L^{2^{*}}\left(\mathbb{R}^{N}\right)}^{2-2^{*}} \leq\left|z_{\varepsilon}\right|_{L^{2^{*}}\left(\mathbb{R}^{N}\right)}^{2} \leq|U|_{L^{2^{*}}\left(\mathbb{R}^{N}\right)}^{2},
$$

equivalently, the equality (15) is valid.

Combining (15) and (16), we obtain that

$$
\begin{aligned}
\left(\frac{\left\|z_{\varepsilon}\right\|_{\sigma}^{2}}{\left|z_{\varepsilon}\right|_{L^{2^{*}}\left(\mathbb{R}^{N}\right)}^{2}}-S_{\sigma}\right) & =\left[\frac{\|\left. U\right|_{\sigma} ^{2}+O\left(\varepsilon^{\frac{N-2}{2}}\right)}{|U|_{L^{2^{*}}\left(\mathbb{R}^{N}\right)}^{2}+O\left(\varepsilon^{\frac{N}{2}}\right)}-\frac{\|\left. U\right|_{\sigma} ^{2}}{|U|_{L^{2^{*}}\left(\mathbb{R}^{N}\right)}^{2}}\right] \\
& =\left[\frac{|U|_{L^{2^{*}}\left(\mathbb{R}^{N}\right)}^{2} O\left(\varepsilon^{\frac{N-2}{2}}\right)-\left.|| U\right|_{\sigma} ^{2} O\left(\varepsilon^{\frac{N}{2}}\right)}{\left(|U|_{L^{2^{*}}\left(\mathbb{R}^{N}\right)}^{2}+O\left(\varepsilon^{\frac{N}{2}}\right)\right)|U|_{L^{*}\left(\mathbb{R}^{N}\right)}^{2}}\right] \\
& =O\left(\varepsilon^{\frac{N-2}{2}}\right) .
\end{aligned}
$$

Step 2 Using the fact that 
$\max _{t \geq 0}\left(\frac{t^{2}}{2} S-\frac{t^{2^{*}}}{2^{*}} T\right)=\frac{1}{N}\left(\frac{S}{T^{\frac{2}{2^{*}}}}\right)^{\frac{N}{2}}, \quad$ forany $\mathrm{S}, \mathrm{T}>0$

and using $\left(F_{1}\right),(3),(4)$, and (15)-(17), we conclude that

$$
\begin{aligned}
\sup _{t \geq 0} \bar{G}(t) & =\frac{1}{N}\left(\frac{\left(e_{1}^{2}+e_{2}^{2}\right) \int_{\Omega}\left(\left|\nabla z_{\varepsilon}\right|^{2}-\sigma \frac{\left(z_{\varepsilon}\right)^{2}}{|x|^{2}}\right)}{\left(\int_{\Omega} F\left(x, e_{1} z_{\varepsilon}, e_{2} z_{\varepsilon}\right) \mathrm{d} x\right)^{\frac{2}{2^{*}}}}\right)^{\frac{N}{2}} \\
& =\frac{1}{N}\left(\frac{\int_{\Omega}\left(\left|\nabla z_{\varepsilon}\right|^{2}-\sigma \frac{\left(z_{\varepsilon}\right)^{2}}{|x|^{2}}\right)}{\left(M_{F}^{\frac{2^{*}}{2}} \int_{\Omega}\left|z_{\varepsilon}\right|^{2^{*}} \mathrm{~d} x\right)^{\frac{2}{2}}}\right)^{\frac{2^{*}}{2}} \\
& \leq \frac{1}{N}\left(\frac{1}{M_{F}}\right)^{N / 2}\left(S_{\sigma}+o\left(\varepsilon^{\frac{N-2}{2}}\right)\right)^{N / 2} \\
& =\frac{1}{N} S_{F}^{\frac{N}{2}}+o\left(\varepsilon^{\frac{N-2}{2}}\right) .
\end{aligned}
$$

Let $d_{0}$ be the positive constant given in Lemma 3.4. We can choose $\varepsilon_{1}>0$, such that for all $0<\left(\alpha\left|g^{+}\right|_{\infty}\right)^{\frac{2}{2-q}}$ $+\left(\beta\left|h^{+}\right|_{\infty}\right)^{\frac{2}{2-q}}<\varepsilon_{1}$, then

$c_{\infty}=\frac{1}{N} S_{F}^{\frac{2}{N}}-d_{0}\left(\left(\alpha\left|g^{+}\right|_{\infty}\right)^{\frac{2}{2-q}}+\left(\beta\left|h^{+}\right|_{\infty}\right)^{\frac{2}{2-q}}>0\right.$.

Using the definitions $E_{\alpha, \beta}$ and $\left(z_{0}, y_{0}\right)$, we obtain

$E_{\alpha, \beta}\left(t z_{0}, y_{0}\right) \leq \frac{t^{2}}{2}\left\|\left(z_{0}, y_{0}\right)\right\|^{2}, \quad(t \geq 0, \alpha>0, \beta>0)$,

which implies that there exists $t_{0} \in(0,1)$ satisfying

$$
\begin{aligned}
& \sup _{0 \leq t \leq t_{0}} E_{\alpha, \beta}\left(t z_{0}, y_{0}\right)<c_{\infty}, \quad \text { for all } \\
& 0<\left(\alpha\left|g^{+}\right|_{\infty}\right)^{\frac{2}{2-q}}+\left(\beta\left|h^{+}\right|_{\infty}\right)^{\frac{2}{2-q}}<\varepsilon_{1}
\end{aligned}
$$

Using the definitions of $E_{\alpha, \beta},\left(z_{0}, y_{0}\right)$, and Step 1, we have

$$
\begin{aligned}
\sup _{t \geq t_{0}} E_{\alpha, \beta}\left(t z_{0}, t y_{0}\right)= & \sup _{t \geq t_{0}}\left(\bar{G}(t)-\frac{t^{q}}{q} D_{\alpha, \beta}\left(z_{0}, y_{0}\right)\right) \\
\leq & \frac{1}{N} I_{F}^{\frac{N}{2}}+O\left(\varepsilon^{\frac{N-2}{2}}\right)-\frac{t_{0}^{q}}{q}\left(\left(e_{1}^{q}\left|g^{+}\right|_{\infty} \alpha\right)\right. \\
& +\left(e_{2}^{q}\left|h^{+}\right|_{\infty} \beta\right) \int_{B_{\varepsilon_{0}}(0)}\left|z_{\varepsilon}\right|^{q} \mathrm{~d} x \\
\leq & \frac{1}{N} I_{F}^{\frac{N}{2}}+O\left(\varepsilon^{\frac{N-2}{2}}\right)-\frac{t_{0}^{q}}{q} m(\alpha+\sigma) \\
& \int_{B_{\varepsilon_{0}}(0)}\left|z_{\varepsilon}\right|^{q} \mathrm{~d} x,
\end{aligned}
$$

where $m=\min \left\{e_{1}^{q}\left|g^{+}\right|_{\infty}, e_{2}^{q}\left|h^{+}\right|_{\infty}\right\}$. Let $0<\varepsilon \leq \varepsilon_{0}^{\frac{\tau-\tau^{\prime}}{\sqrt{\sigma}}}$, then

$$
\begin{aligned}
\int_{B_{\varepsilon_{0}}(0)}\left|z_{\varepsilon}\right|^{q} \mathrm{~d} x & =\int_{B_{\varepsilon_{0}}(0)} \frac{\varepsilon^{\frac{q(N-2)}{4}}}{\left(\varepsilon|x|^{\tau^{\prime} / \sqrt{\bar{\sigma}}}+|x|^{\tau / \sqrt{\bar{\sigma}}}\right)^{q \sqrt{\bar{\sigma}}}} \\
& \geq \int_{B_{\varepsilon_{0}}(0)} \frac{\varepsilon^{\frac{q(N-2)}{4}}}{\left(2 \varepsilon_{0}^{\frac{\tau}{\sqrt{\bar{\sigma}}}}\right)^{q \sqrt{\bar{\sigma}}}} \\
& =d_{1}\left(N, q, \sigma, \varepsilon_{0}\right) \varepsilon^{\frac{q(N-2)}{4}} .
\end{aligned}
$$

Combining relations (21) and (28), for all

$$
\varepsilon=\left(\left(\alpha\left|g^{+}\right|_{\infty}\right)^{\frac{2}{2-q}}+\left(\beta\left|h^{+}\right|_{\infty}\right)^{\frac{2}{2-q}}\right)^{\frac{2}{N-2}} \in\left(0, \varrho_{0}^{\frac{\tau-\tau^{\prime}}{\sqrt{\sigma}}}\right)
$$

, we have

$$
\begin{gathered}
\sup _{t \geq t_{0}} E_{\alpha, \beta}\left(t z_{0}, t y_{0}\right) \leq \frac{1}{N} I_{F}^{\frac{N}{2}}+O\left(\alpha\left|g^{+}\right|_{\infty}\right)^{\frac{2}{2-q}} \\
\left.+\left(\beta\left|h^{+}\right|_{\infty}\right)^{\frac{2}{2-q}}\right)-\frac{t_{0}^{q}}{q} m d_{1}(\alpha+\sigma) .
\end{gathered}
$$

we can choose $\varepsilon_{2}>0$, such that for all $0<\left(\alpha\left|g^{+}\right|_{\infty}\right)^{\frac{2}{2-q}}+\left(\beta\left|h^{+}\right|_{\infty}\right)^{\frac{2}{2-q}}<\varepsilon_{2}$, we have

$$
\begin{aligned}
& O\left(\left(\alpha\left|g^{+}\right|_{\infty}\right)^{\frac{2}{2-q}}+\left(\beta\left|h^{+}\right|_{\infty}\right)^{\frac{2}{2-q}}\right)-\frac{t_{0}^{q}}{q} m d_{1}(\alpha+\sigma) \\
& \quad<-d_{0}\left(\left(\alpha\left|g^{+}\right|_{\infty}\right)^{\frac{2}{2-q}}+\left(\beta\left|h^{+}\right|_{\infty}\right)^{\frac{2}{2-q}}\right) .
\end{aligned}
$$

If we set $\omega^{*}=\min \left\{\varepsilon_{1}, \varepsilon_{0}^{N-2}, \varepsilon_{2}\right\}$ and $\varepsilon=\left(\left(\alpha\left|g^{+}\right|_{\infty}\right)^{\frac{2}{2-q}}+\right.$ $\left.\left(\beta\left|h^{+}\right|_{\infty}\right)^{\frac{2}{2-q}}\right)^{\frac{2}{N-2}}$, then for $0<\left(\alpha\left|g^{+}\right|_{\infty}\right)^{\frac{2}{2-q}}+\left(\beta\left|h^{+}\right|_{\infty}\right)^{\frac{2}{2-q}}$ $<\omega^{*}$ :

$\sup _{t \geq 0} E_{\alpha, \beta}\left(t z_{0}, t y_{0}\right)<c_{\infty}$

Finally, we prove that $\vartheta_{\alpha, \beta}^{-}<c_{\infty}$ for all $0<\left(\alpha\left|g^{+}\right|_{\infty}\right)^{\frac{2}{2-q}}+$ $\left(\beta\left|h^{+}\right|_{\infty}\right)^{\frac{2}{2-q}}<\omega^{*}$. Recall that $\left(z_{0}, y_{0}\right)=\left(e_{1} z_{\varepsilon}, e_{2} z_{\varepsilon}\right)$. It is easy to see that

$\int_{\Omega} F\left(x, z_{0}, y_{0}\right) \mathrm{d} x>0, \quad D_{\alpha, \beta}\left(z_{0}, y_{0}\right)>0$.

Using Lemma 2.6, and from the definition of $\vartheta_{\alpha, \beta}^{-}$and (30), there exists $t_{0}>0$, such that $\left(t_{0} z_{0}, t_{0} y_{0}\right) \in \mathcal{N}_{\alpha, \beta}^{-}$and

$\vartheta_{\alpha, \beta}^{-} \leq E_{\alpha, \beta}\left(t_{0} z_{0}, t_{0} y_{0}\right) \leq \sup _{t \geq 0} E_{\alpha, \beta}\left(t z_{0}, t y_{0}\right)<c_{\infty}$.

This completes the proof.

Now, we establish the existence of a local minimum of $E_{\alpha, \beta}$ on $\mathcal{N}_{\alpha, \beta}^{-}$.

Theorem 3.8 If $0<\left(\alpha\left|g^{+}\right|_{\infty}\right)^{\frac{2}{2-q}}+\left(\beta\left|h^{+}\right|_{\infty}\right)^{\frac{2}{2-q}}<\omega_{0}^{*}$, $\left(F_{1}\right)-\left(F_{3}\right)$ hold, then $E_{\alpha, \beta}$ has a minimizer $\left(z_{0}^{-}, y_{0}^{-}\right)$in $\mathcal{N}_{\alpha, \beta}^{-}$and 
(i) $E_{\alpha, \beta}\left(z_{0}^{-}, y_{0}^{-}\right)=\vartheta_{\alpha, \beta}^{-}$,

(ii) $\left(z_{0}^{-}, y_{0}^{-}\right)$is a positive solution of problem (1), where

$$
\omega_{0}^{*}=\min \left\{\omega^{*},\left(\frac{q}{2}\right)^{\frac{2}{2-q}} \omega\right\} \text {. }
$$

Proof By Lemma 3.2(ii), there is a (PS $)_{\vartheta_{\alpha, \beta}^{-}}$sequence $\left\{\left(z_{n}, y_{n}\right)\right\} \subset \mathcal{N}_{\alpha, \beta}^{-}$in $Q$ for $E_{\alpha, \beta}$ for all $\alpha, \beta \in \Theta_{\omega_{0}}$. From Lemmas 3.6, 3.7 and 2.5(ii), for $\alpha, \beta \in \Theta_{\omega^{*}}, E_{\alpha, \beta}$ satisfies (PS) ${\vartheta_{\alpha, \beta}^{-}}$condition and $\vartheta_{\alpha, \beta}^{-}>0$. Since $E_{\alpha, \beta}$ is coercive on $\mathcal{N}_{\alpha, \beta}^{-}$, we get that $\left(z_{n}, y_{n}\right)$ is bounded in $Q$. Therefore, there exists a subsequence still denoted by $\left(z_{n}, y_{n}\right)$ and $\left(z_{0}^{-}, y_{0}^{-}\right) \in \mathcal{N}_{\alpha, \beta}^{-}$, such that $\left(z_{n}, y_{n}\right) \rightarrow\left(z_{0}^{-}, y_{0}^{-}\right)$strongly in $Q$ and $E_{\alpha, \beta}\left(z_{0}^{-}, y_{0}^{-}\right)=\vartheta_{\alpha, \beta}^{-}>0$ for all $\alpha, \beta \in \Theta_{\omega_{1}}$. Finally, by the same arguments as in the proof of Theorem 3.3, for all $\alpha, \beta \in \Theta_{\omega_{1}}$, we have that $\left(z_{0}^{-}, y_{0}^{-}\right)$is a positive solution of problem (1).

Now, we complete the proof of Theorems 1.2 and 1.3; by Theorem 3.3, we obtain that for all $\alpha, \beta \in \Theta_{\omega}$, problem (1) has a positive solution $\left(z_{0}^{+}, y_{0}^{+}\right) \in \mathcal{N}_{\alpha, \beta}^{+}$. On the other hand, from Theorem 3.8, we get the second positive solution $\left(z_{0}^{-}, y_{0}^{-}\right) \in \mathcal{N}_{\alpha, \beta}^{-} \quad$ for $\quad$ all $\quad \alpha, \beta \in \Theta_{\omega_{1}}$. Since $\mathcal{N}_{\alpha, \beta}^{+} \cap \mathcal{N}_{\alpha, \beta}^{-}=\emptyset$, this implies that $\left(z_{0}^{+}, y_{0}^{+}\right)$and $\left(z_{0}^{-}, y_{0}^{-}\right)$are distinct. This completes the proof of Theorems 1.2 and 1.3.

Acknowledgements The authors are grateful to the referee for some valuable comments that led to an improvement of the manuscript.

Open Access This article is distributed under the terms of the Creative Commons Attribution 4.0 International License (http://crea tivecommons.org/licenses/by/4.0/), which permits unrestricted use, distribution, and reproduction in any medium, provided you give appropriate credit to the original author(s) and the source, provide a link to the Creative Commons license, and indicate if changes were made.

\section{References}

1. Alves, C.O., de Moraes Fillho, D.C., Miyagaki, O.H.: Multiple solutions for an elliptic system on bounded or unbounded domains. Nonlinear Anal. 56, 555-568 (2004)
2. Brown, K.J., Wu, T.F.: A semilinear elliptic system involving nonlinear boundary condition and sign changing weight function. J. Math. Anal. Appl. 337, 1326-1336 (2008)

3. Brezis, H., Lieb, E.: A relation between pointwise convergence of functions and convergece of functionals. Proc. Am. Math. Soc. 88, 486-490 (1983)

4. Brown, K.J., Zhang, Y.: The Nehari manifold for a semilinear elliptic problem with a sign changing weight function. J. Differ. Equ. 193, 481-499 (2003)

5. Caffarelli, L., Kohn, R., Nirenberg, L.: First order interpolation inequality with weights. Compos. Math. 53, 259-275 (1984)

6. Chou, K.S., Chu, C.W.: On the best constant for a weighted Sobolev-Hardi inequality. Commun. J. Lond. Math. Soc. 48, 137-151 (1993)

7. Chu, C.M., Tang, C.L.: Existence and multiplicity of positive solutions for semilinear elliptic system with Sobolev critical exponent. Nonlinear Anal. 71, 5118-5130 (2009)

8. Ekeland, I.: On the variational principle. J. Math. Anal. Appl. 17, 324353 (1974)

9. Ferrero, A., Gazzola, F.: Existence of solutions for singular critical growth semilinear elliptic equations. J. Differ. Equ. 177, 494-522 (2001)

10. de Filho, D.C.M., Souto, M.A.S.: System of $p$-Laplacian equations involving homogeneous nonlinearities with critical Sobolev exponent degrees. Commun. Partial Differ. Equ. 24, 1537-1553 (1999)

11. Hsu, T.S.: Multiple positive solutions for a critical quasilinear elliptic system with concave-convex nonlinearities. Nonlinear Anal. 71, 2688-2698 (2009)

12. Hsu, T.S.: Multiple positive solutions for semilinear elliptic equations involving multi-singular inverse square potentials and concave-convex nonlinearities. Nonlinear Anal. 74, 3703-3715 (2011)

13. Hsu, T.S.: On the elliptic problem involving multisingular inverse square potentials and concave-convex nonlinearities. Abstr. Appl. Anal. 408307, 1-22 (2011)

14. Hsu, T.S., Lin, H.L.: Multiple solutions for singular elliptic equations with weighted Hardy terms and critical Sobolev-Hardy exponents. Proc. R. Soc. Edinb. Sect. A 140, 617-633 (2010)

15. Wu, T.F.: On semilinear elliptic equations involving concaveconvex nonlinearities and sign-changing weight function. J. Math. Anal. Appl. 318, 253-270 (2006) 